Mathematical Modelling and Analysis

Volume 19 Number 4, September 2014, 450-468 http://dx.doi.org/10.3846/13926292.2014.955893

(C) Vilnius Gediminas Technical University, 2014
Publisher: Taylor\&Francis and VGTU

http://www.tandfonline.com/TMMA

Print ISSN: 1392-6292

Online ISSN: 1648-3510

\title{
Efficient General Linear Methods of High Order with Inherent Quadratic Stability*
}

\author{
Michał Braś ${ }^{b, c}$ and Zdzisław Jackiewicz ${ }^{a, b}$ \\ ${ }^{a}$ School of Mathematical \& Statistical Sciences, Arizona State University \\ Tempe, Arizona 85287 \\ ${ }^{b}$ AGH University of Science and Technology, Faculty of Applied Mathematics \\ al. Mickiewicza 30, 30-059 Krakow, Poland \\ c Jagiellonian University, Faculty of Mathematics and Computer Science \\ ul. prof. S. Łojasiewicza 6, 30-348 Krakow, Poland \\ E-mail(corresp.): bras@agh.edu.pl \\ E-mail: jackiewicz@asu.edu
}

Received September 5, 2013; revised July 3, 2014; published online September 1, 2014

\begin{abstract}
We search for general linear methods with $s$ internal stages and $r=s+1$ external stages of order $p=s+1$ and stage order $q=s$. We require that stability function of these methods has only two non-zero roots. This is achieved by imposing the so-called inherent quadratic stability conditions. Examples of such general linear methods which are $A$ - and $L$-stable up to the order $p=8$ and stage order $q=p-1$ are derived.
\end{abstract}

Keywords: general linear methods, order and stage order, $A$ - and $L$-stability, inherent quadratic stability.

AMS Subject Classification: 65L05; 65L20.

\section{Introduction}

Consider the initial value problem for an autonomous system of ordinary differential equations of the following form

$$
y^{\prime}(t)=f(y(t)), \quad y\left(t_{0}\right)=y_{0},
$$

$t \in\left[t_{0}, T\right]$, where the function $f: \mathbb{R}^{m} \rightarrow \mathbb{R}^{m}$ is sufficiently smooth and $y_{0} \in \mathbb{R}^{m}$ is a given initial value.

The general linear methods (GLMs) are given by the abscissa vector $\mathbf{c}=$ $\left[c_{1}, \ldots, c_{s}\right]^{T}$ and the coefficient matrices $\mathbf{A}=\left[a_{i j}\right], \mathbf{U}=\left[u_{i j}\right], \mathbf{B}=\left[b_{i j}\right]$, and

\footnotetext{
* The work of Michał Braś was supported by the National Science Center under grant DEC2011/01/N/ST1/02672 and by the Polish Ministry of Science and Higher Education.
} 
$\mathbf{V}=\left[v_{i j}\right]$, where $\mathbf{A} \in \mathbb{R}^{s \times s}, \mathbf{U} \in \mathbb{R}^{s \times r}, \mathbf{B} \in \mathbb{R}^{r \times s}, \mathbf{V} \in \mathbb{R}^{r \times r}$. On the uniform grid $t_{n}=t_{0}+n h, n=0,1, \ldots, N, N h=T-t_{0}$, these methods have the form

$$
\begin{aligned}
Y_{i}^{[n]} & =h \sum_{j=1}^{s} a_{i j} f\left(Y_{j}^{[n]}\right)+\sum_{j=1}^{r} u_{i j} y_{j}^{[n-1]}, \quad i=1,2, \ldots, s, \\
y_{i}^{[n]}=h \sum_{j=1}^{s} b_{i j} f\left(Y_{j}^{[n]}\right)+\sum_{j=1}^{r} v_{i j} y_{j}^{[n-1]}, & i=1,2, \ldots, r,
\end{aligned}
$$

$n=1,2, \ldots, N$. Here $Y_{i}^{[n]}, i=1,2, \ldots, s$, are approximations of stage order $q$ to $y\left(t_{n-1}+c_{i} h\right)$, and $y_{i}^{[n]}, i=1,2, \ldots, r$, are approximations of order $p$ to the linear combinations of the derivatives of $y$ at the grid point $t_{n}$. Different representations of GLMs are discussed in the monograph [7].

We assume that the matrices $\mathbf{A}$ and $\mathbf{V}$ have the form

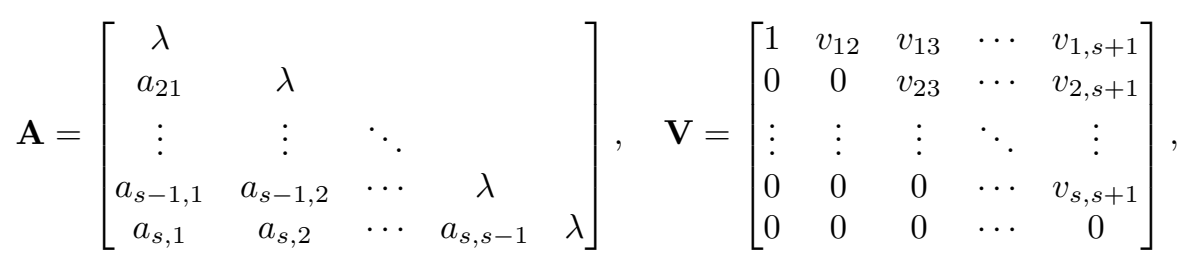

so that the resulting methods have low implementation costs and are zerostable. In [6] the authors investigated the class of Nordsieck methods of order $p=r=s+1$ and stage order $q=p$ or $q=p-1$. Since the order of these methods is $p=s+1$ these formulas are more efficient than diagonally implicit multistage integration methods (DIMSIMs) with parameters $p=q=r=s$ investigated in $[8,9,10,11,13,22,24]$, or GLM with inherent Runge-Kutta stability (IRKS) property with parameters $p=q=r-1=s-1$ investigated in $[12,14,22,25,26]$. However, direct approach to construction of these methods presented in [6] was limited to order $p=2,3$, and 4 .

In [2] (compare also $[3,4,15,16,17])$ the approach to construct Nordsieck methods via inherent quadratic stability (IQS) was presented. It means that some algebraic conditions on coefficient matrices of the methods are imposed to guarantee that the stability function has only two non-zero roots. Therefore we can limit the linear stability analysis to the quadratic part of the stability function.

In present paper we combine both results from [6] and [2] to overcome technical difficulties and construct the families of $A$ - and $L$-stable methods with parameters $p=q+1=r=s+1$ and IQS property. The order of constructed methods is $p=2,3, \ldots, 8$.

The paper is organized as follow: in Section 2 we recall the order and stage order conditions derived in [6] together with representation formulas for methods of order $p=s+1$ and stage order $q=p-1$ and some aspects of error propagation analysis. In Section 3 we present the concept of inherent quadratic stability, and present results obtained in [2] and [3]. In Section 4 we combine the results presented in previous two sections to construct $A$ - and $L$ - stable methods of order $p=s+1$ and stage order $q=p-1$ with inherent quadratic 
property and give examples of such methods of order $p=2,3, \ldots, 8$. Section 5 contains some concluding remarks.

\section{Order Conditions and Error Propagation}

\subsection{Order conditions and representation formulas}

We assume (compare, for example, [22]) that the components $y_{i}^{[n-1]}$ of the input vector for the step from $t_{n-1}$ to $t_{n}$ approximate the linear combinations of the scaled derivatives $h^{k} y^{(k)}\left(t_{n-1}\right)$ up to the order $p=s+1$, i.e.,

$$
y_{i}^{[n-1]}=\sum_{k=0}^{s+1} q_{i k} h^{k} y^{(k)}\left(t_{n-1}\right)+O\left(h^{s+2}\right),
$$

$i=1,2, \ldots, r$, for some constants $q_{i k}$. The GLM (1.1) is said to have stage order $q=s$ if from (2.1) follows, that the components $Y_{i}^{[n]}$ of the vector of internal stages $Y^{[n]}$ approximate $y\left(t_{n-1}+c_{i} h\right)$ up to the stage order $q$, i.e.,

$$
Y_{i}^{[n]}=y\left(t_{n-1}+c_{i} h\right)+O\left(h^{q+1}\right)
$$

$i=1,2, \ldots, s$, where $q=s$. The GLM (1.1) is said to have order $p=s+1$ if from (2.1) follows, that the components $y_{i}^{[n]}$ of the input vector $y^{[n]}$ for the next step from $t_{n}$ to $t_{n+1}$ satisfy $(2.1)$ with $n-1$ replaced by $n$, i.e.,

$$
y_{i}^{[n]}=\sum_{k=0}^{s+1} q_{i k} h^{k} y^{(k)}\left(t_{n}\right)+O\left(h^{s+2}\right),
$$

$i=1,2, \ldots, r$, with the same constants $q_{i k}$.

Similarly as in $[8,9,22]$ we can write the stage order and order conditions corresponding to $(2.1),(2.2)$, and (2.3) in a compact form using functions of a complex variable $z$. We define the vectors $\mathbf{q}_{i}$ by

$$
\mathbf{q}_{i}=\left[\begin{array}{lll}
q_{1, i} & \cdots & q_{r, i}
\end{array}\right]^{T}, \quad i=0,1, \ldots, r,
$$

and the vector functions $\widetilde{\mathbf{w}}=\widetilde{\mathbf{w}}(z)$ and $\mathbf{w}=\mathbf{w}(z)$ of a complex variable $z$ by

$$
\widetilde{\mathbf{w}}(z)=\sum_{i=0}^{s} \mathbf{q}_{i} z^{i}, \quad \mathbf{w}(z)=\sum_{i=0}^{s+1} \mathbf{q}_{i} z^{i}, \quad z \in \mathbb{C} .
$$

Now, the order conditions can be written in more convenient for use in symbolic manipulation software (Mathematica, Maple) form. The GLM (1.1) has stage order $q=s$ or $q=s+1$ and order $p=s+1$ if and only if

$$
\begin{aligned}
& e^{\mathbf{c} z}=z \mathbf{A} e^{\mathbf{c} z}+\mathbf{U} \widetilde{\mathbf{w}}(z)+O\left(z^{s+1}\right) \\
& e^{z} \mathbf{w}(z)=z \mathbf{B} e^{\mathbf{c} z}+\mathbf{V} \mathbf{w}(z)+O\left(z^{s+2}\right),
\end{aligned}
$$

where $e^{\mathbf{c} z}=\left[\begin{array}{lll}e^{c_{1} z} & \cdots & e^{c_{s} z}\end{array}\right]^{T}$. 
We are interested in the class of GLMs (1.1) for which the vectors $\mathbf{q}_{0}, \mathbf{q}_{1}, \ldots, \mathbf{q}_{s}$ defined in (2.4) take the special form $\mathbf{q}_{0}=\mathbf{e}_{1}, \mathbf{q}_{1}=\mathbf{e}_{2}, \ldots, \mathbf{q}_{s}=\mathbf{e}_{s+1}$, where $\mathbf{e}_{1}, \mathbf{e}_{2}, \ldots, \mathbf{e}_{s+1}$ is the canonical basis in the space $\mathbb{R}^{s+1}$. Then the vector of external approximations take the form

$$
y^{[n]}=z\left(t_{n}, h\right)+\mathbf{q}_{s+1} h^{s+1} y^{(s+1)}\left(t_{n}\right)+O\left(h^{s+2}\right),
$$

where $z(t, h)$ is the Nordsieck vector defined by

$$
z(t, h)=\left[y(t), h y^{\prime}(t), \ldots, h^{s} y^{(s)}(t)\right]^{T} \in \mathbb{R}^{(s+1) m} .
$$

We will also call the class of resulting GLMs the Nordsieck methods.

Following presentation in [6] we introduce the matrices

$$
\mathbf{C}=\left[\begin{array}{llll}
\mathbf{e} & \mathbf{c} & \cdots & \frac{\mathbf{c}^{s}}{s !}
\end{array}\right], \quad \mathbf{K}=\left[\begin{array}{ccccc}
0 & 1 & 0 & \cdots & 0 \\
0 & 0 & 1 & \cdots & 0 \\
\vdots & \vdots & \vdots & \ddots & \vdots \\
0 & 0 & 0 & \cdots & 1 \\
0 & 0 & 0 & \cdots & 0
\end{array}\right],
$$

where $\mathbf{C} \in \mathbb{R}^{s \times(s+1)}$ and $\mathbf{K} \in \mathbb{R}^{(s+1) \times(s+1)}$.

The Nordsieck method has stage order $q=s$ if and only if

$$
\mathbf{U}=\mathbf{C}-\mathbf{A C K},
$$

while the Nordsieck method has order $p=s+1$ if and only if

$$
\mathbf{V}=\mathbf{E}-\mathbf{B C K},
$$

where $\mathbf{E}=\exp (\mathbf{K})$, and

$$
\mathbf{V} \mathbf{q}_{s+1}=\mathbf{q}_{s+1}+\mathbf{t}_{s+1}-\mathbf{B} \frac{\mathbf{c}^{s}}{s !}
$$

(compare [6]).

The relation (2.6) is usually inverted in order to express the matrix $\mathbf{B}$ in terms of $\mathbf{V}$ and $\mathbf{c}$. We partition the coefficient matrices $\mathbf{B}$ and $\mathbf{V}$ and the matrix $\mathbf{E}$ into the form

$$
\mathbf{B}=\left[\begin{array}{c}
b^{T} \\
\hline B
\end{array}\right], \quad \mathbf{V}=\left[\begin{array}{c|c}
1 & v^{T} \\
\hline 0 & V
\end{array}\right], \quad \mathbf{E}=\left[\begin{array}{c|c}
1 & \mathbf{t}_{s}^{T} \\
\hline 0 & E
\end{array}\right],
$$

where $b \in \mathbb{R}^{s}, B \in \mathbb{R}^{s \times s}, v \in \mathbb{R}^{s}, V \in \mathbb{R}^{s \times s}, \mathbf{t}_{s} \in \mathbb{R}^{s}$, and $E \in \mathbb{R}^{s \times s}$, and define the matrix

$$
C=\left[\begin{array}{llll}
\mathbf{e} & \mathbf{c} & \cdots & \frac{\mathbf{c}^{s-1}}{(s-1) !}
\end{array}\right] \in \mathbb{R}^{s \times s} .
$$

Assuming that components of vector $\mathbf{c}$ are distinct we write the inverted relation $(2.6)$ as

$$
b^{T}=\left(\mathbf{t}_{s}^{T}-v^{T}\right) C^{-1},
$$

and

$$
B=(E-V) C^{-1}
$$




\subsection{Error propagation analysis}

Similarly as in [6] we assume that the components of the input vector $y^{[n-1]}$ at the grid point $t_{n-1}$ have the form

$$
\begin{aligned}
y_{i}^{[n-1]}= & \sum_{k=0}^{p} q_{i k} h^{k} y^{(k)}\left(t_{n-1}\right)-\beta_{i} h^{p+1} y^{(p+1)}\left(t_{n-1}\right) \\
& -\delta_{i} h^{p+1} \frac{\partial f}{\partial y}\left(y\left(t_{n-1}\right)\right) y^{(p)}\left(t_{n-1}\right)+O\left(h^{p+2}\right),
\end{aligned}
$$

$i=1,2, \ldots, r$, with $\beta_{1}=\delta_{1}=0$, and that the components of the vector of internal approximations $Y^{[n]}$ have the form

$$
Y_{i}^{[n]}=y\left(t_{n-1}+c_{i} h\right)-\xi_{i} h^{p} y^{(p)}\left(t_{n-1}\right)+O\left(h^{p+1}\right),
$$

$i=1,2, \ldots, s$. Than the components of the vector $y^{[n]}$ at the next grid point $t_{n}=t_{n-1}+h$ satisfy the relations

$$
\begin{aligned}
y_{1}^{[n]}= & \sum_{k=0}^{p} q_{1, k} h^{k} y^{(k)}\left(t_{n}\right)-E h^{p+1} y^{(p+1)}\left(t_{n}\right) \\
& -F h^{p+1} \frac{\partial f}{\partial y}\left(y\left(t_{n}\right)\right) y^{(p)}\left(t_{n}\right)+O\left(h^{p+2}\right), \\
y_{i}^{[n]}= & \sum_{k=0}^{p} q_{i k} h^{k} y^{(k)}\left(t_{n}\right)-\beta_{i} h^{p+1} y^{(p+1)}\left(t_{n}\right) \\
& -\delta_{i} h^{p+1} \frac{\partial f}{\partial y}\left(y\left(t_{n}\right)\right) y^{(p)}\left(t_{n}\right)+O\left(h^{p+2}\right),
\end{aligned}
$$

$i=2,3, \ldots, r$, for the same constants $\beta_{i}$ and $\delta_{i}$ if

$$
E=\sum_{k=0}^{p} \frac{q_{1, k}}{(p+1-k) !}-\frac{b^{T} \mathbf{c}^{p}}{p !}+v^{T} \widetilde{\beta}, \quad F=b^{T} \xi+v^{T} \widetilde{\delta},
$$

where

$$
\widetilde{\beta}=(I-V)^{-1}\left(\eta-\frac{B \mathbf{c}^{p}}{p !}\right), \quad \xi=\frac{\mathbf{c}^{p}}{p !}-\frac{\mathbf{A} \mathbf{c}^{p-1}}{(p-1) !}-\mathbf{U} \mathbf{q}_{p}, \quad \widetilde{\delta}=(I-V)^{-1} B \xi,
$$

and the vector $\eta$ is defined by

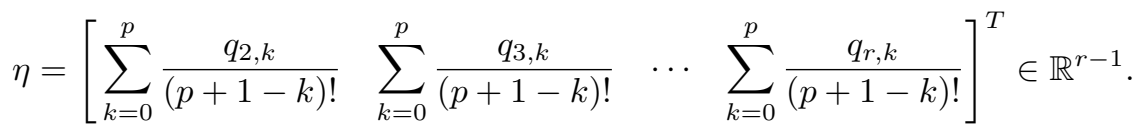

The matrices $B, V$ and vectors $b^{T}$ and $v^{T}$ were defined in (2.8), and vectors $\beta$ and $\delta$ are partitioned as

$$
\beta=\left[\begin{array}{l}
0 \\
\widetilde{\beta}
\end{array}\right] \in \mathbb{R}^{r}, \quad \delta=\left[\begin{array}{l}
0 \\
\widetilde{\delta}
\end{array}\right] \in \mathbb{R}^{r},
$$


where $\widetilde{\beta} \in \mathbb{R}^{r-1}, \widetilde{\delta} \in \mathbb{R}^{r-1}$.

Since the local discretization error le $\left(t_{n}\right)$ of the method (1.1) is defined by

$$
\mathrm{le}\left(t_{n}\right)=E h^{p+1} y^{(p+1)}\left(t_{n}\right)+F h^{p+1} \frac{\partial f}{\partial y}\left(y\left(t_{n}\right)\right) y^{(p)}\left(t_{n}\right)
$$

we search for an approximations of terms $h^{p+1} y^{(p+1)}\left(t_{n}\right)$ and $h^{p+1} \frac{\partial f}{\partial y}\left(y\left(t_{n}\right)\right) \times$ $y^{(p)}\left(t_{n}\right)$

$$
\begin{aligned}
& h^{p+1} y^{(p+1)}\left(t_{n}\right)=h \sum_{j=1}^{s} \varphi_{j} f\left(Y_{j}^{[n]}\right)+\sum_{j=1}^{r} \psi_{j} y_{j}^{[n-1]}+O\left(h^{p+2}\right), \\
& h^{p+1} \frac{\partial f}{\partial y}\left(y\left(t_{n}\right)\right) y^{(p)}\left(t_{n}\right)=h \sum_{j=1}^{s} \bar{\varphi}_{j} f\left(Y_{j}^{[n]}\right)+\sum_{j=1}^{r} \bar{\psi}_{j} y_{j}^{[n-1]}+O\left(h^{p+2}\right),
\end{aligned}
$$

where

$$
\varphi=\left[\begin{array}{llll}
\varphi_{1} & \varphi_{2} & \cdots & \varphi_{s}
\end{array}\right]^{T} \in \mathbb{R}^{s}, \quad \psi=\left[\begin{array}{llll}
\psi_{1} & \psi_{2} & \cdots & \psi_{r}
\end{array}\right]^{T} \in \mathbb{R}^{r},
$$

and

$$
\bar{\varphi}=\left[\begin{array}{llll}
\bar{\varphi}_{1} & \bar{\varphi}_{2} & \cdots & \bar{\varphi}_{s}
\end{array}\right]^{T} \in \mathbb{R}^{s}, \quad \bar{\psi}=\left[\begin{array}{llll}
\bar{\psi}_{1} & \bar{\psi}_{2} & \cdots & \bar{\psi}_{r}
\end{array}\right]^{T} \in \mathbb{R}^{r} .
$$

It was proved in [6] that vectors $\varphi, \psi$, and $\bar{\varphi}, \bar{\psi}$ satisfy the systems of equations

$$
\left\{\begin{array}{l}
\frac{\varphi^{T} \mathbf{c}^{k-1}}{(k-1) !}+\psi^{T} \mathbf{q}_{k}=0, \quad k=0,1, \ldots, p, \\
\frac{\varphi^{T} \mathbf{c}^{p}}{p !}-\psi^{T} \beta=1, \quad \varphi^{T} \xi+\psi^{T} \delta=0,
\end{array}\right.
$$

and

$$
\left\{\begin{array}{l}
\frac{\bar{\varphi}^{T} \mathbf{c}^{k-1}}{(k-1) !}+\bar{\psi}^{T} \mathbf{q}_{k}=0, \quad k=0,1, \ldots, p, \\
\frac{\bar{\varphi}^{T} \mathbf{c}^{p}}{p !}-\bar{\psi}^{T} \beta=0, \quad \bar{\varphi}^{T} \xi+\bar{\psi}^{T} \delta=-1,
\end{array}\right.
$$

where we use the convention that $(-1) !=\infty$.

\section{Inherent Quadratic Stability}

The stability properties of GLM (1.1) with respect to linear test equation [19]

$$
y^{\prime}=\xi y,
$$

$\xi \in \mathbb{C}$, are defined by stability function

$$
p(w, z)=\operatorname{det}(w \mathbf{I}-\mathbf{M}(z)),
$$

where $z=\xi h$ and stability matrix $\mathbf{M}(z)$ is given by

$$
\mathbf{M}(z)=\mathbf{V}+z \mathbf{B}(\mathbf{I}-z \mathbf{A})^{-1} \mathbf{U} .
$$


Stability function $p(w, z)$ is a rational function. To simplify analysis we multiply stability function $p(w, z)$ by $(1-\lambda w)^{s}$ and we work with stability function denoted by the same symbol $p$ which is a polynomial with respect to $w$ whose coefficients are polynomials with respect to $z$.

We recall (compare, for example [22]) that the method is said to be absolutely stable in $z$ if $p(w, z)$ has roots of modulus less than 1 . The set of all $z \in \mathbb{C}$ such that methods is absolutely stable is called the region of absolute stability. The method is $A$-stable if its region of absolute stability contains a negative half plane.

The method is said to be $L$-stable, if it is $A$-stable and

$$
\lim _{z \rightarrow \infty} \rho(\mathbf{M}(z))=0,
$$

where $\rho(\mathbf{M}(z))$ is the spectral radius of stability matrix $\mathbf{M}(z)$. For the discussion of algebraic stability property of general linear methods we refer to $[5,18,20,21,22]$.

To investigate Nordsieck methods with so called quadratic stability function, i.e., stability function with only two nonzero roots, we introduce equivalence relation between matrices of the same dimensions. We say that the two matrices $\mathbf{D}$ and $\mathbf{E}$ are equivalent, denoted by $\mathbf{D} \equiv \mathbf{E}$, if they are equal except for the first two rows (compare [17,22]).

We assume that there exists a matrix $\mathbf{X} \in \mathbb{R}^{(s+1) \times(s+1)}$ such that

$$
\mathbf{B A} \equiv \mathbf{X B}, \quad \mathbf{B U} \equiv \mathbf{X V}-\mathbf{V X} .
$$

Then (compare $[2,3,4,15,16])$ the stability function $p(w, z)$ of GLM assumes the quadratic form:

$$
p(w, z)=w^{s-1}\left(w^{2}(1-\lambda z)^{s}-p_{1}(z) w+p_{0}(z)\right),
$$

where coefficients $p_{1}(z)$ and $p_{0}(z)$ are polynomials of the form

$$
\begin{aligned}
& p_{1}(z)=1+p_{11} z+p_{12} z^{2}+\cdots+p_{1 s} z^{s}, \\
& p_{0}(z)=p_{01} z+p_{02} z^{2}+\cdots+p_{0 s} z^{s} .
\end{aligned}
$$

It was demonstrated in [3] that for GLM with $p=q+1=s+1=r$ the most general matrix $\mathbf{X}$ satisfying conditions (3.1) is of the form

$$
\mathbf{X}=\left[\begin{array}{ccccc|c}
x_{1,1} & x_{1,2} & x_{1,3} & \cdots & x_{1, r-1} & x_{1, r} \\
x_{2,1} & x_{2,2} & x_{2,3} & \cdots & x_{2, r-1} & x_{2, r} \\
\hline 0 & 1 & 0 & \cdots & 0 & x_{3, r} \\
0 & 0 & 1 & \cdots & 0 & x_{4, r} \\
\vdots & \vdots & \vdots & \ddots & \vdots & \vdots \\
0 & 0 & 0 & \cdots & 1 & x_{r, r}
\end{array}\right]
$$

\section{Search for Methods of Order $p=s+1=r$ and Stage Order $q=p-1$}

In this section we will describe the search for Nordsieck methods of order $p=$ $s+1=r$ and stage order $q=p-1$. It will be always assumed that vector $\mathbf{c}$ 
is given in advance. We begin with applying order representation formulas (2.5), (2.9) and (2.10) corresponding to order $p=q=s=r-1$. Taking into account the form of matrix $\mathbf{X}$ given by (3.3), we solve IQS conditions (3.1) with respect to $x_{i r}, i=3,4, \ldots, r, a_{i j}, i=2,3 \ldots, s, j=1,2, \ldots, i$, and $v_{i, j}, i=2,3, \ldots, r-1, j=i+1, i+2, \ldots, r$. We solve the remaining order condition (2.7) with respect to $v_{1 r}, q_{i r}, i=2,3, \ldots, r$. We fix $q_{1 r}=0$, so $y_{1}^{[n]}=y\left(t_{n}\right)+O\left(h^{s+2}\right)$. Finally, we solve the system (compare (3.2))

$$
p_{1 s}=0, \quad p_{0 s}=0
$$

to ensure that $A$-stable methods are also $L$-stable. The remaining free parameters are used to find families of $A$-stable methods.

In the following subsections we will use the described above procedure to search for methods of order $p=3,4, \ldots, 8$. Case $p=2$ is an exception, since the stability polynomial has quadratic form without any additional assumptions. We also present sets of constants $E$ and $F$, vectors $\widetilde{\beta}, \widetilde{\delta}, \xi$ and $\eta$ for derived examples of methods. It was observed in [6], that systems (2.13) and (2.14) consist of $s+4$ equations in $2 s+1$ unknowns and have solutions for $s \geq 3$. For $s \geq 4$ we require that both terms in one before last equation of system (2.13) and last equation of system (2.14) have the same contributions, i.e.,

$$
\frac{\varphi^{T} \mathbf{c}^{p}}{p !}=\frac{1}{2}, \quad-\psi^{T} \beta=\frac{1}{2}, \quad \bar{\varphi}^{T} \xi=-\frac{1}{2}, \quad \bar{\psi}^{T} \delta=-\frac{1}{2} .
$$

For $s \geq 5$ we require that (4.2) is satisfied and, in addition, that the sums of squares of elements of vectors $\varphi$ and $\psi$ or $\bar{\varphi}$ and $\bar{\psi}$, i.e.,

$$
\varphi_{1}^{2}+\cdots+\varphi_{s}^{2}+\psi_{1}^{2}+\cdots+\psi_{r}^{2} \quad \text { or } \quad \bar{\varphi}_{1}^{2}+\cdots+\bar{\varphi}_{s}^{2}+\bar{\psi}_{1}^{2}+\cdots+\bar{\psi}_{r}^{2}
$$

are minimal (compare [1]).

\subsection{Construction of methods with $p=r=2, q=s=1$}

The polynomial of the method with parameters $p=r=2$ and $s=q=1$ assumes quadratic form without any additional conditions. Similarly as in $[6]$ we assume that $\mathbf{q}_{2}=[0,0]^{T}$, so $y^{[n]}=z\left(t_{n}, h\right)+O\left(h^{3}\right)$. We derive coefficient matrices $\mathbf{U}$ and $\mathbf{B}$ from representation formulas, and solve the remaining order condition with respect to $v_{12}$ and $c_{1}$, and obtain the one-parameter family of the Nordsieck methods depending on $\lambda$. The coefficients of these methods are $\mathbf{c}=1$ and

$$
\left[\begin{array}{c|c}
\mathbf{A} & \mathbf{U} \\
\hline \mathbf{B} & \mathbf{V}
\end{array}\right]=\left[\begin{array}{c|cc}
\lambda & 1 & 1-\lambda \\
\hline \frac{1}{2} & 1 & \frac{1}{2} \\
1 & 0 & 0
\end{array}\right] .
$$

We apply Schur theorem [23] (compare also [22] for illustrative examples) and obtain that these methods are $A$-stable if $\lambda \geq 1 / 2$. There are no $L$-stable methods in this family. For $\lambda=\frac{1}{2}$ we have

$$
\left[\begin{array}{c|c}
\mathbf{A} & \mathbf{U} \\
\hline \mathbf{B} & \mathbf{V}
\end{array}\right]=\left[\begin{array}{c|cc}
\frac{1}{2} & 1 & \frac{1}{2} \\
\hline \frac{1}{2} & 1 & \frac{1}{2} \\
1 & 0 & 0
\end{array}\right] .
$$


For this method we have

$$
\eta=\frac{1}{2}, \quad \widetilde{\beta}=0, \quad \xi=0, \quad \widetilde{\delta}=0, \quad E=-\frac{1}{12}, \quad F=0 .
$$

This method was derived in [6] and is presented here for the sake of completeness.

\subsection{Construction of methods with $p=r=3, q=s=2$}

We fix in advance the abscissa vector $\mathbf{c}=[0,1]^{T}$. After applying order and IQS conditions as described at the beginning of the section, we solve system (4.1) with respect to $\lambda$ and $v_{1,2}$. This system has two solutions $\lambda=\frac{1}{6}(4-\sqrt{6})$, $v_{1,2}=\frac{1}{18}(-11+4 \sqrt{6})$ and $\lambda=\frac{1}{6}(4+\sqrt{6}), v_{1,2}=\frac{1}{18}(-11-4 \sqrt{6})$.

We verify using Schur criterion that the method corresponding only to the second solution leads to $A$-stable, hence also $L$-stable, method. The quadratic part of the stability function is given by

$$
p(w, z)=\left(1-\frac{1}{6}(4+\sqrt{6}) z\right)^{2} w^{2}+\left(-1+\frac{1}{9}(5+4 \sqrt{6}) z\right) w-\frac{1}{9}(2+\sqrt{6}) z .
$$

We note, that the method with the same value of $\lambda$ parameter was constructed in [6] via quadratic stability, but these methods differ due to another approach and another choice of free parameters. The coefficient of this method are

$$
\begin{gathered}
\mathbf{A}=\left[\begin{array}{cc}
\frac{4+\sqrt{6}}{6} & 0 \\
1 & \frac{4+\sqrt{6}}{6}
\end{array}\right], \quad \mathbf{U}=\left[\begin{array}{ccc}
1 & -\frac{4+\sqrt{6}}{6} & 0 \\
1 & -\frac{4+\sqrt{6}}{6} & -\frac{1+\sqrt{6}}{6}
\end{array}\right], \\
\mathbf{B}=\left[\begin{array}{cc}
\frac{194+9 \sqrt{6}}{108} & \frac{5(16+3 \sqrt{6})}{108} \\
-\frac{1+\sqrt{6}}{6} & \frac{7+\sqrt{6}}{6} \\
-1 & 1
\end{array}\right], \quad \mathbf{V}=\left[\begin{array}{ccc}
1 & -\frac{11+4 \sqrt{6}}{18} & -\frac{26+15 \sqrt{6}}{108} \\
0 & 0 & -\frac{1+\sqrt{6}}{6} \\
0 & 0 & 0
\end{array}\right],
\end{gathered}
$$

and the vector $\mathbf{q}_{3}=\left[\begin{array}{lll}0 & \frac{1+\sqrt{6}}{6} & -\frac{1}{2}\end{array}\right]^{T}$. For this method we have

$$
\begin{gathered}
\eta=\left[\begin{array}{ll}
\frac{2+\sqrt{6}}{6} & 0
\end{array}\right]^{T}, \quad \widetilde{\beta}=\left[\begin{array}{ll}
\frac{1+\sqrt{6}}{6} & -\frac{1}{6}
\end{array}\right]^{T}, \quad \xi=\left[\begin{array}{ll}
\frac{10+5 \sqrt{6}}{36} & \frac{1-\sqrt{6}}{36}
\end{array}\right]^{T}, \\
\widetilde{\delta}=\left[\begin{array}{ll}
\frac{1-\sqrt{6}}{36} & \frac{-3-2 \sqrt{6}}{12}
\end{array}\right]^{T}, \quad E=\frac{-79-30 \sqrt{6}}{216}, \quad F=\frac{57+23 \sqrt{6}}{108} .
\end{gathered}
$$

\subsection{Construction of methods with $p=r=4, q=s=3$}

We fix in advance the abscissa vector $\mathbf{c}=\left[0, \frac{1}{2}, 1\right]^{T}$. After applying order and IQS conditions we solve system (4.1) with respect to $v_{1,2}$ and $v_{1,3}$ and obtain the one-parameter family of methods depending on $\lambda$. By the Schur theorem, the $A$ - and $L$ - stable methods are those for which $0.398633 \leq \lambda \leq 0.519543$ or $1.36276 \leq \lambda \leq 1.56234$. 
The coefficients of $L$-stable method corresponding to $\lambda=\frac{1}{2}$ are

$$
\begin{array}{cc}
\mathbf{A}=\left[\begin{array}{rrr}
\frac{1}{2} & 0 & 0 \\
\frac{1}{2} & \frac{1}{2} & 0 \\
\frac{1}{2} & \frac{1}{2} & \frac{1}{2}
\end{array}\right], & \mathbf{U}=\left[\begin{array}{rrrr}
1 & -\frac{1}{2} & 0 & 0 \\
1 & -\frac{1}{2} & -\frac{1}{8} & -\frac{1}{24} \\
1 & -\frac{1}{2} & -\frac{1}{4} & -\frac{7}{48}
\end{array}\right], \\
\mathbf{B}=\left[\begin{array}{rrr}
0 & 1 & \frac{1}{4} \\
-\frac{2}{3} & \frac{1}{3} & \frac{4}{3} \\
2 & -6 & 4 \\
4 & -8 & 4
\end{array}\right], & \mathbf{V}=\left[\begin{array}{rrrr}
1 & -\frac{1}{4} & -\frac{1}{4} & -\frac{1}{12} \\
0 & 0 & -\frac{1}{2} & -\frac{5}{24} \\
0 & 0 & 0 & -\frac{1}{4} \\
0 & 0 & 0 & 0
\end{array}\right],
\end{array}
$$

and the vector $\mathbf{q}_{4}=\left[\begin{array}{llll}0, & \frac{1}{12}, & \frac{1}{6}, & -\frac{1}{2}\end{array}\right]^{T}$. For this method we have

$$
\begin{gathered}
\eta=\left[\begin{array}{lll}
\frac{1}{8} & \frac{1}{3} & 0
\end{array}\right]^{T}, \quad \widetilde{\beta}=\left[\begin{array}{lll}
-\frac{1}{96} & \frac{7}{32} & -\frac{7}{48}
\end{array}\right]^{T}, \\
\xi=\left[\begin{array}{lll}
\frac{1}{24} & \frac{13}{384} & -\frac{1}{24}
\end{array}\right]^{T}, \quad \widetilde{\delta}=\left[\begin{array}{lll}
\frac{3}{32} & -\frac{7}{32} & -\frac{13}{48}
\end{array}\right]^{T}, \\
E=-\frac{257}{5760}, \quad F=\frac{89}{1152}, \\
\varphi=\left[\begin{array}{llll}
37 & -11 & 13
\end{array}\right]^{T}, \quad \psi=\left[\begin{array}{llll}
0 & -39 & -\frac{15}{2} & -\frac{41}{8}
\end{array}\right]^{T}, \\
\bar{\varphi}=\left[\begin{array}{lll}
33 & -15 & 9
\end{array}\right]^{T}, \quad \bar{\psi}=\left[\begin{array}{llll}
0 & -27 & -\frac{3}{2} & -\frac{21}{8}
\end{array}\right]^{T} .
\end{gathered}
$$

\subsection{Construction of methods with $p=r=5, q=s=4$}

We fix in advance the abscissa vector $\mathbf{c}=\left[0, \frac{1}{3}, \frac{2}{3}, 1\right]^{T}$. After applying order and IQS conditions we solve system (4.1) with respect to $v_{1,3}$ and $v_{1,4}$ and obtain the two-parameter family of methods depending on $\lambda$ and $v_{1,2}$.

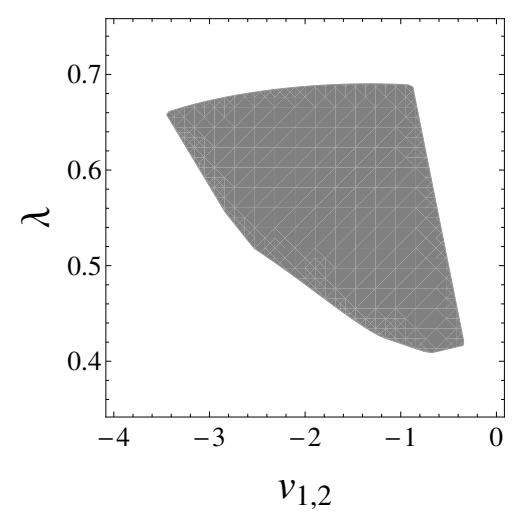

Figure 1. $A$ - and $L$-stable methods of stage order $q=4$ and order $p=5$.

The range of parameters $\lambda$ and $v_{1,2}$ for which the methods are $A$ - and $L$ stable is plotted in Figure 1. Again, this result was obtained by applying the 
Schur criterion. The coefficients of $L$-stable method corresponding to $\lambda=\frac{1}{2}$ and $v_{12}=-2$ are

$$
\begin{gathered}
\mathbf{A}=\left[\begin{array}{cccc}
\frac{1}{2} & 0 & 0 & 0 \\
\frac{1}{3} & \frac{1}{2} & 0 & 0 \\
\frac{1}{3} & \frac{1}{3} & \frac{1}{2} & 0 \\
\frac{1}{3} & \frac{1}{3} & \frac{1}{3} & \frac{1}{2}
\end{array}\right], \quad \mathbf{U}=\left[\begin{array}{rrrrr}
1 & -\frac{1}{2} & 0 & 0 & 0 \\
1 & -\frac{1}{2} & -\frac{1}{9} & -\frac{7}{324} & -\frac{5}{1944} \\
1 & -\frac{1}{2} & -\frac{2}{9} & -\frac{13}{162} & -\frac{1}{54} \\
1 & -\frac{1}{2} & -\frac{1}{3} & -\frac{19}{108} & -\frac{13}{216}
\end{array}\right], \\
\mathbf{B}=\left[\begin{array}{rrrr}
-\frac{11}{1440} & \frac{1229}{1440} & -\frac{151}{480} & \frac{79}{32} \\
\frac{49}{24} & -\frac{143}{24} & \frac{67}{24} & \frac{17}{8} \\
\frac{13}{4} & -\frac{9}{4} & -\frac{33}{4} & \frac{29}{4} \\
-18 & 63 & -72 & 27 \\
-27 & 81 & -81 & 27
\end{array}\right], \quad \mathbf{V}\left[\begin{array}{rrrrr}
1 & -2 & -\frac{2207}{1080} & -\frac{6773}{6480} & -\frac{8387}{23328} \\
0 & 0 & -1 & -\frac{23}{27} & -\frac{187}{648} \\
0 & 0 & 0 & -\frac{2}{3} & -\frac{31}{108} \\
0 & 0 & 0 & 0 & -\frac{1}{3} \\
0 & 0 & 0 & 0 & 0
\end{array}\right],
\end{gathered}
$$

and the vector $\mathbf{q}_{5}=\left[\begin{array}{lllll}0, & -\frac{1}{24}, & \frac{1}{18}, & \frac{25}{108}, & -\frac{1}{2}\end{array}\right]^{T}$. For this method we have

$$
\begin{aligned}
& \eta=\left[\begin{array}{llll}
-\frac{1}{30} & \frac{7}{72} & \frac{43}{108} & 0
\end{array}\right]^{T}, \quad \widetilde{\beta}=\left[\begin{array}{lllll}
-\frac{901}{5832} & -\frac{181}{1620} & \frac{8}{27} & -\frac{5}{36}
\end{array}\right]^{T}, \\
& \xi=\left[-\frac{1}{48},-\frac{977}{87480},-\frac{413}{174960},-\frac{23}{3240}\right]^{T}, \quad \widetilde{\delta}=\left[\frac{3533}{29160}, \frac{587}{3240},-\frac{17}{72},-\frac{37}{108}\right]^{T} \text {, } \\
& E=\frac{7543}{29160}, \quad F=-\frac{62693}{233280}, \\
& \varphi=\left[\begin{array}{llll}
-\frac{790050555}{12191264} & \frac{1462288365}{12191264} & -\frac{958072095}{12191264} & \frac{150626385}{12191264}
\end{array}\right]^{T}, \\
& \psi=\left[\begin{array}{lllll}
0 & \frac{33801975}{3047816} & \frac{329445}{6095632} & \frac{56353475}{12191264} & \frac{1235750}{1142931}
\end{array}\right]^{T}, \\
& \bar{\varphi}=\left[\begin{array}{llll}
-\frac{316456200}{1717511} & \frac{506673900}{1717511} & -\frac{592565760}{1717511} & \frac{449613180}{1717511}
\end{array}\right]^{T}, \\
& \bar{\psi}=\left[\begin{array}{lllll}
0 & -\frac{47265120}{1717511} & -\frac{223460640}{1717511} & -\frac{121273860}{1717511} & -\frac{48800640}{1717511}
\end{array}\right]^{T} .
\end{aligned}
$$

\subsection{Construction of methods with $p=r=6, q=s=5$}

We fix in advance the abscissa vector $\mathbf{c}=\left[0, \frac{1}{4}, \frac{1}{2}, \frac{3}{4}, 1\right]^{T}$. After applying order and IQS conditions we solve system (4.1) with respect to $v_{1,4}$ and $v_{1,5}$ and obtain the three-parameter family of methods depending on $\lambda, v_{1,2}$ and $v_{1,3}$.

We use the Schur criterion to find $A$-, and hence, $L$-stable methods among this family. In Figure 2 we plotted the range of parameters $\lambda$ and $v_{1,2}$ for some chosen values of parameter $v_{1,3}$.

Coefficients and the vector $\mathbf{q}_{6}$ of the $L$-stable method corresponding to $\lambda=\frac{1}{2}, v_{12}=-\frac{3}{2}, v_{13}=-2$ are

$$
\mathbf{q}_{6}=\left[\begin{array}{llllll}
0 & \frac{1}{80} & -\frac{47}{960} & \frac{5}{128} & \frac{17}{64} & -\frac{1}{2}
\end{array}\right]^{T}
$$



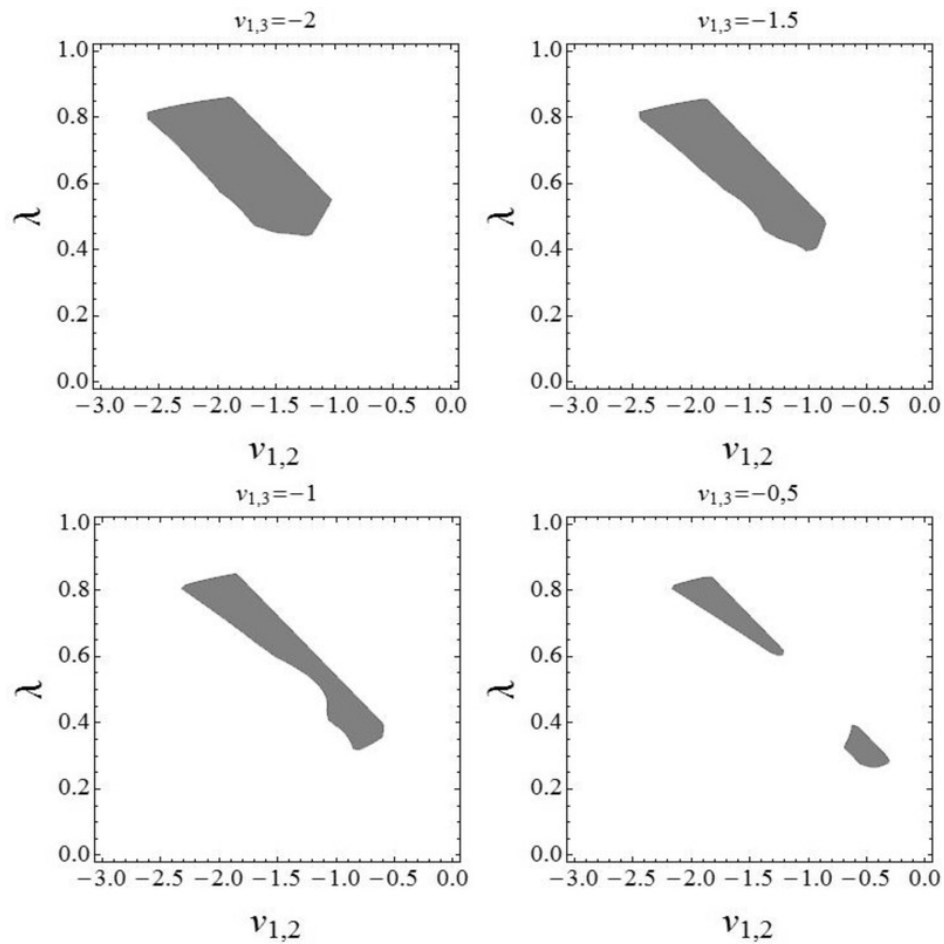

Figure 2. $A$ - and $L$-stable methods of stage order $q=5$ and order $p=6$

$$
\begin{aligned}
& \mathbf{A}=\left[\begin{array}{ccccc}
\frac{1}{2} & 0 & 0 & 0 & 0 \\
\frac{1}{4} & \frac{1}{2} & 0 & 0 & 0 \\
\frac{1}{4} & \frac{1}{4} & \frac{1}{2} & 0 & 0 \\
\frac{1}{4} & \frac{1}{4} & \frac{1}{4} & \frac{1}{2} & 0 \\
\frac{1}{4} & \frac{1}{4} & \frac{1}{4} & \frac{1}{4} & \frac{1}{2}
\end{array}\right], \quad \mathbf{U}=\left[\begin{array}{rrrrrr}
1 & -\frac{1}{2} & 0 & 0 & 0 & 0 \\
1 & -\frac{1}{2} & -\frac{3}{32} & -\frac{5}{384} & -\frac{7}{6144} & -\frac{3}{40960} \\
1 & -\frac{1}{2} & -\frac{3}{16} & -\frac{19}{384} & -\frac{13}{1536} & -\frac{133}{122880} \\
1 & -\frac{1}{2} & -\frac{9}{32} & -\frac{7}{64} & -\frac{57}{2048} & -\frac{163}{30720} \\
1 & -\frac{1}{2} & -\frac{3}{8} & -\frac{37}{192} & -\frac{25}{384} & -\frac{1013}{61440}
\end{array}\right], \\
& \mathbf{B}=\left[\begin{array}{rrrrrrr}
\frac{17}{10} & -\frac{343}{60} & \frac{37}{3} & -\frac{859}{60} & \frac{17}{2} \\
\frac{527}{60} & -\frac{512}{15} & \frac{1149}{20} & -\frac{1609}{30} & \frac{338}{15} \\
-15 & 49 & -37 & -17 & 20 \\
-20 & 16 & 88 & -144 & 60 \\
192 & -832 & 1344 & -960 & 256 \\
256 & -1024 & 1536 & -1024 & 256
\end{array}\right], \\
& \mathbf{V}=\left[\begin{array}{rrrrrrrr}
1 & -\frac{3}{2} & -2 & -\frac{1363}{960} & -\frac{293}{480} & -\frac{34703}{184320} \\
0 & 0 & -\frac{3}{2} & -\frac{115}{64} & -\frac{237}{256} & -\frac{20533}{61440} \\
0 & 0 & 0 & -\frac{9}{8} & -\frac{191}{192} & -\frac{17}{48} \\
0 & 0 & 0 & 0 & -\frac{3}{4} & -\frac{1}{3} \\
0 & 0 & 0 & 0 & 0 & -\frac{3}{8} \\
0 & 0 & 0 & 0 & 0 & 0
\end{array}\right] .
\end{aligned}
$$


For this method we have

$$
\begin{gathered}
E=-\frac{335521}{7741440}, \quad F=\frac{48703}{1105920}, \quad \eta=\left[\begin{array}{lllll}
\frac{1}{72} & -\frac{13}{320} & \frac{31}{384} & \frac{83}{192} & 0
\end{array}\right]^{T}, \\
\widetilde{\beta}=\left[\begin{array}{llllll}
\frac{33491}{122880} & -\frac{1867}{12288} & -\frac{4049}{23040} & \frac{43}{128} & -\frac{13}{96}
\end{array}\right]^{T}, \\
\xi=\left[\begin{array}{llllll}
\frac{1}{160} & \frac{14339}{5898240} & \frac{221}{368640} & \frac{269}{393216} & \frac{13}{11520}
\end{array}\right]^{T}, \\
\widetilde{\delta}=\left[\begin{array}{llllll}
-\frac{17657}{368640} & \frac{7063}{61440} & \frac{5627}{23040} & -\frac{31}{128} & -\frac{73}{192}
\end{array}\right]^{T}, \\
\psi=\left[\begin{array}{llllll}
0 & -408.3249 & -561.5087 & -300.3998 & -89.08654 & -19.28257
\end{array}\right]^{T}, \\
\bar{\varphi}=\left[\begin{array}{llllll}
409.7825 & -1051.314 & 84.34111 & 657.1231 & 337.3543
\end{array}\right]^{T}, \\
\bar{\psi}=\left[\begin{array}{llllll}
0 & -437.2873 & -609.5387 & -331.1821 & -101.4490 & -22.76820
\end{array}\right]^{T} .
\end{gathered}
$$

\subsection{Construction of methods with $p=r=7, q=s=6$}

We fix in advance the abscissa vector $\mathbf{c}=\left[0, \frac{1}{5}, \frac{2}{5}, \frac{3}{5}, \frac{4}{5}, 1\right]^{T}$. After applying order and IQS conditions we solve system (4.1) with respect to $v_{1,5}$ and $v_{1,6}$ and obtain a four-parameters family of methods depending on $\lambda, v_{1,2}, v_{1,3}$ and $v_{1,4}$.

We use the Schur criterion to find $A$-, and hence, $L$-stable methods among this family. In Figure 3 we plotted the range of parameters $\lambda$ and $v_{1,2}$ for some chosen values of parameters $v_{1,3}$ and $v_{1,4}$.

The coefficients of the $L$-stable method corresponding to $\lambda=\frac{1}{2}, v_{12}=-\frac{11}{4}$, $v_{13}=-5$, and $v_{14}=-4$ are

$$
\begin{aligned}
\mathbf{A}=\left[\begin{array}{ccccccc}
\frac{1}{2} & 0 & 0 & 0 & 0 & 0 \\
\frac{1}{5} & \frac{1}{2} & 0 & 0 & 0 & 0 \\
\frac{1}{5} & \frac{1}{5} & \frac{1}{2} & 0 & 0 & 0 \\
\frac{1}{5} & \frac{1}{5} & \frac{1}{5} & \frac{1}{2} & 0 & 0 \\
\frac{1}{5} & \frac{1}{5} & \frac{1}{5} & \frac{1}{5} & \frac{1}{2} & 0 \\
\frac{1}{5} & \frac{1}{5} & \frac{1}{5} & \frac{1}{5} & \frac{1}{5} & \frac{1}{2}
\end{array}\right], \\
\mathbf{U}=\left[\begin{array}{lllllllll}
1 & -\frac{1}{2} & 0 & 0 & 0 & 0 & 0 \\
1 & -\frac{1}{2} & -\frac{2}{25} & -\frac{13}{1500} & -\frac{3}{5000} & -\frac{23}{750000} & -\frac{7}{5625000} \\
1 & -\frac{1}{2} & -\frac{4}{25} & -\frac{1}{30} & -\frac{17}{3750} & -\frac{173}{375000} & -\frac{211}{5625000} \\
1 & -\frac{1}{2} & -\frac{6}{25} & -\frac{37}{500} & -\frac{3}{200} & -\frac{1709}{750000} & -\frac{173}{625000} \\
1 & -\frac{1}{2} & -\frac{8}{25} & -\frac{49}{375} & -\frac{22}{625} & -\frac{1333}{187500} & -\frac{323}{281250} \\
1 & -\frac{1}{2} & -\frac{2}{5} & -\frac{61}{300} & -\frac{41}{600} & -\frac{861}{50000} & -\frac{781}{225000}
\end{array}\right],
\end{aligned}
$$



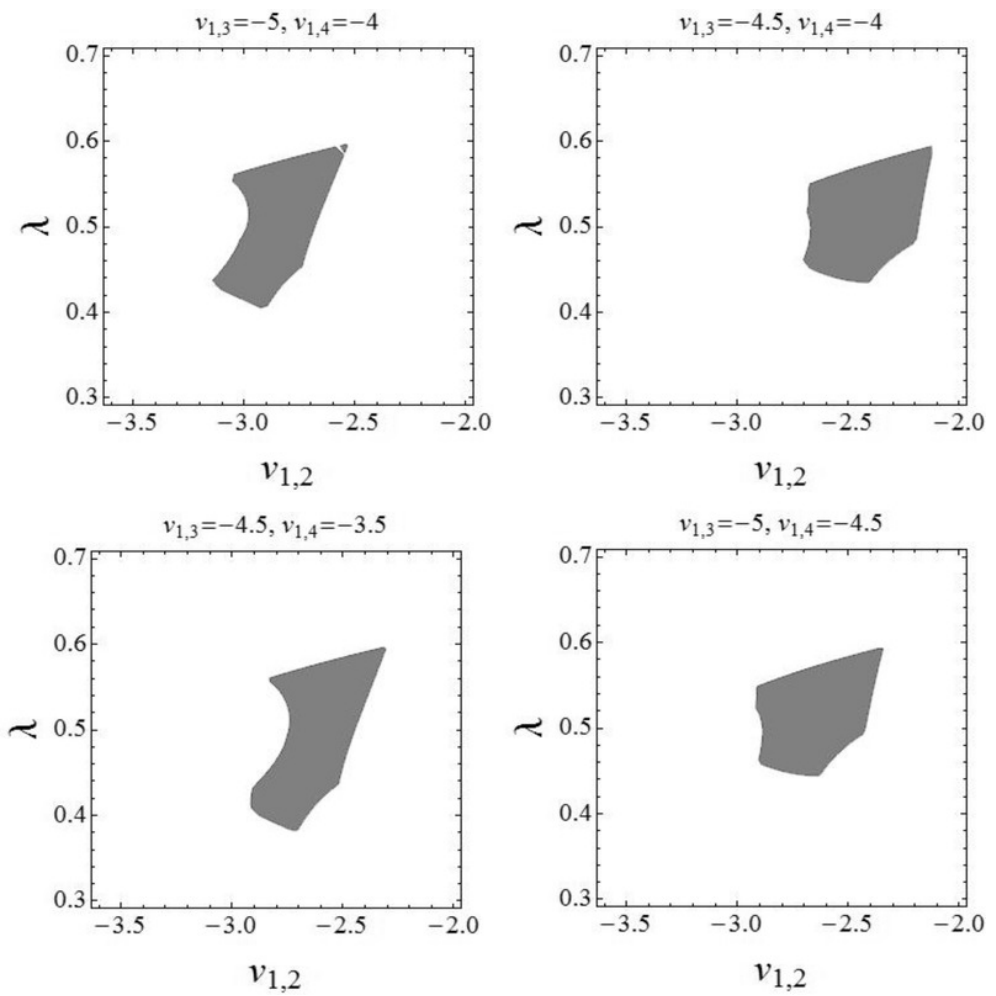

Figure 3. $A$ - and $L$-stable methods of stage order $q=6$ and order $p=7$.

$$
\mathbf{B}=\left[\begin{array}{rrrrrr}
\frac{155423}{8400} & -\frac{426313}{4800} & \frac{1117093}{6300} & -\frac{2791631}{16800} & \frac{91367}{1680} & \frac{4913}{576} \\
\frac{24551}{1440} & -\frac{8341}{160} & \frac{4553}{720} & \frac{110633}{720} & -\frac{34081}{160} & \frac{25663}{288} \\
-\frac{18083}{144} & \frac{94255}{144} & -\frac{104425}{72} & \frac{123985}{72} & -\frac{156715}{144} & \frac{41423}{144} \\
\frac{3175}{24} & -\frac{3875}{8} & \frac{5275}{12} & \frac{3475}{12} & -\frac{4975}{8} & \frac{5875}{24} \\
\frac{875}{6} & -\frac{125}{3} & -\frac{4250}{3} & \frac{9125}{3} & -\frac{14375}{6} & \frac{2000}{3} \\
-2500 & 13125 & -27500 & 28750 & -15000 & 3125 \\
-3125 & 15625 & -31250 & 31250 & -15625 & 3125
\end{array}\right],
$$

$$
\mathbf{V}=\left[\begin{array}{rrrrccc}
1 & -\frac{11}{4} & -5 & -4 & -\frac{5706761}{3150000} & -\frac{11783987}{21000000} & -\frac{987643}{7875000} \\
0 & 0 & -2 & -\frac{226}{75} & -\frac{6113}{3000} & -\frac{97747}{112500} & -\frac{568241}{2250000} \\
0 & 0 & 0 & -\frac{8}{5} & -\frac{197}{100} & -\frac{1041}{1000} & -\frac{10619}{28125} \\
0 & 0 & 0 & 0 & -\frac{6}{5} & -\frac{163}{150} & -\frac{399}{1000} \\
0 & 0 & 0 & 0 & 0 & -\frac{4}{5} & -\frac{109}{300} \\
0 & 0 & 0 & 0 & 0 & 0 & -\frac{2}{5} \\
0 & 0 & 0 & 0 & 0 & 0 & 0
\end{array}\right],
$$

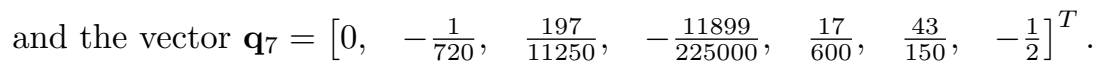


For this method we have

$$
\begin{gathered}
E=-\frac{6616699}{9843750}, F=\frac{212246243}{315000000}, \eta=\left[\begin{array}{llll}
-\frac{1}{840}, \frac{189}{10000},-\frac{1253}{28125}, \frac{7}{100}, \frac{34}{75}, 0
\end{array}\right]^{T}, \\
\widetilde{\beta}=\left[\begin{array}{lllllll}
-\frac{599873}{7500000} & \frac{5287531}{15750000} & -\frac{10853}{75000} & -\frac{16393}{75000} & \frac{9}{25} & -\frac{2}{15}
\end{array}\right]^{T}, \\
\xi=\left[\begin{array}{lllllll}
-\frac{1}{1440} & \frac{1291057}{4725000000} & \frac{1379509}{2362500000} & \frac{797879}{1575000000} & \frac{721619}{2362500000} & \frac{4189}{37800000}
\end{array}\right]^{T}, \\
\widetilde{\delta}=\left[\begin{array}{lllllll}
\frac{25366541}{315000000} & -\frac{427313}{1312500} & \frac{15113}{150000} & \frac{128531}{450000} & -\frac{49}{200} & -\frac{121}{300}
\end{array}\right]^{T}, \\
\varphi=\left[\begin{array}{lllllll}
-558.140 & 1936.402 & 915.807 & -1827.848 & -3139.624 & 3228.07
\end{array}\right]^{T}, \\
\psi=\left[\begin{array}{lllllll}
0 & -554.6663 & -373.2648 & -392.3351 & -216.645 & -72.15556 & -17.22618
\end{array}\right]^{T}, \\
\bar{\varphi}=\left[\begin{array}{lllllll}
5816.926 & -11880.34 & 1763.567 & 10471.44 & 4416.253 & -8100.762
\end{array}\right]^{T}, \\
\bar{\psi}=\left[\begin{array}{lllllll}
0 & -2487.088 & -44.46463 & 848.8418 & 593.3305 & 204.5261 & 48.54273
\end{array}\right]^{T} .
\end{gathered}
$$

\subsection{Construction of methods with $p=r=8, q=s=7$}

We fix in advance the abscissa vector $\mathbf{c}=\left[0, \frac{1}{6}, \frac{1}{3}, \frac{1}{2}, \frac{2}{3}, \frac{5}{6}, 1\right]^{T}$. After applying order and IQS conditions we solve system (4.1) with respect to $v_{1,6}$ and $v_{1,7}$ and obtain a five-parameter family of methods depending on $\lambda, v_{1,2}, v_{1,3}, v_{1,4}$, and $v_{1,5}$.

We use the Schur criterion to find $A$-, and hence, $L$-stable methods among this family. In Figure 4 we plotted the range of parameters $\lambda$ and $v_{1,2}$ for some chosen values of parameters $v_{1,3}, v_{1,4}$ and $v_{1,5}$.

Coefficients and the vector $\mathbf{q}_{8}$ of $L$-stable method corresponding to $\lambda=\frac{1}{2}$, $v_{12}=-\frac{12}{5}, v_{13}=-\frac{7}{2}, v_{14}=-2$, and $v_{15}=0$ are

$$
\begin{gathered}
\mathbf{q}_{8}=\left[\begin{array}{llllllllll}
0 & -\frac{5}{4032} & -\frac{3151}{1088640} & & \frac{983}{46656} & & -\frac{4301}{77760} & \frac{1}{48} & \frac{65}{216} & -\frac{1}{2}
\end{array}\right]^{T}, \\
\mathbf{A}=\left[\begin{array}{ccccccc}
\frac{1}{2} & 0 & 0 & 0 & 0 & 0 & 0 \\
\frac{1}{6} & \frac{1}{2} & 0 & 0 & 0 & 0 & 0 \\
\frac{1}{6} & \frac{1}{6} & \frac{1}{2} & 0 & 0 & 0 & 0 \\
\frac{1}{6} & \frac{1}{6} & \frac{1}{6} & \frac{1}{2} & 0 & 0 & 0 \\
\frac{1}{6} & \frac{1}{6} & \frac{1}{6} & \frac{1}{6} & \frac{1}{2} & 0 & 0 \\
\frac{1}{6} & \frac{1}{6} & \frac{1}{6} & \frac{1}{6} & \frac{1}{6} & \frac{1}{2} & 0 \\
\frac{1}{6} & \frac{1}{6} & \frac{1}{6} & \frac{1}{6} & \frac{1}{6} & \frac{1}{6} & \frac{1}{2}
\end{array}\right]
\end{gathered}
$$



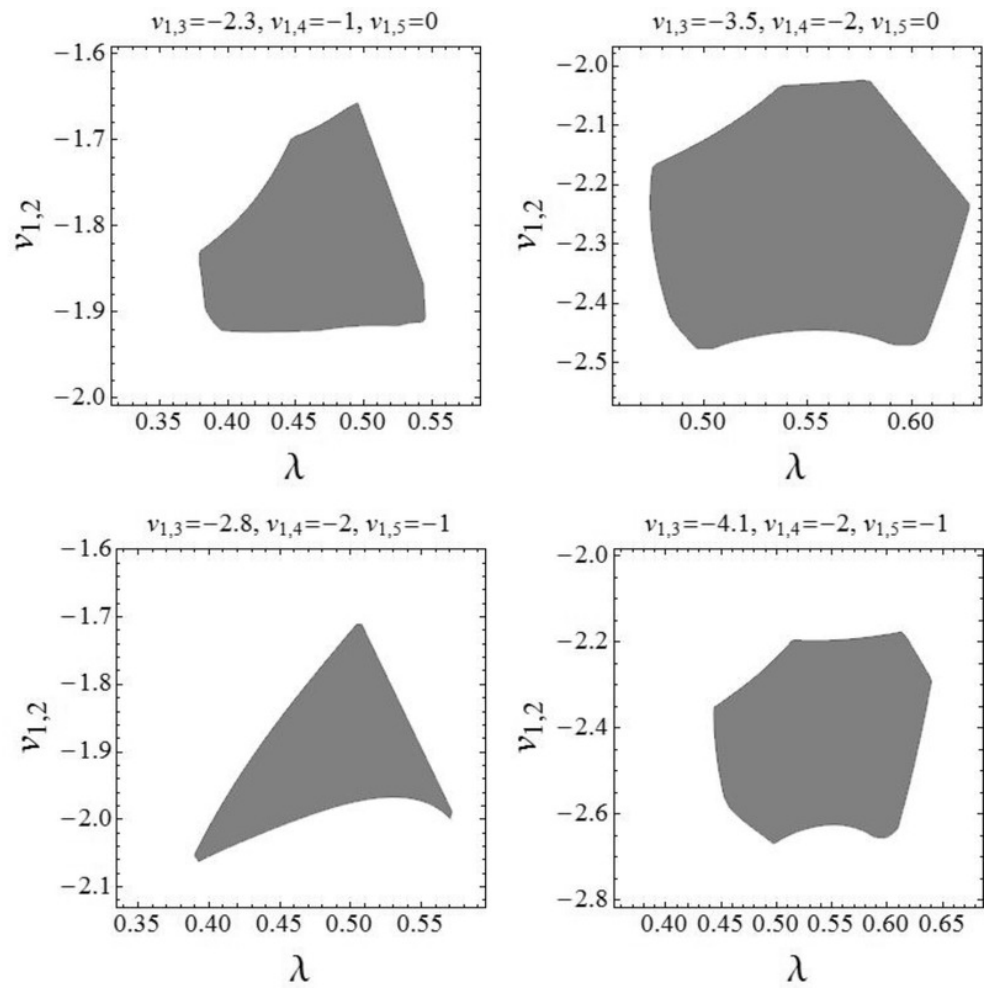

Figure 4. $A$ - and $L$-stable methods of stage order $q=7$ and order $p=8$.

$$
\mathbf{U}=\left[\begin{array}{cccccccc}
1 & -\frac{1}{2} & 0 & 0 & 0 & 0 & 0 & 0 \\
1 & -\frac{1}{2} & -\frac{5}{72} & -\frac{1}{162} & -\frac{11}{31104} & -\frac{7}{466560} & -\frac{17}{33592320} & -\frac{1}{70543872} \\
1 & -\frac{1}{2} & -\frac{5}{36} & -\frac{31}{1296} & -\frac{7}{2592} & -\frac{71}{311040} & -\frac{259}{16796160} & -\frac{1223}{1410877440} \\
1 & -\frac{1}{2} & -\frac{5}{24} & -\frac{23}{432} & -\frac{31}{3456} & -\frac{1057}{933120} & -\frac{427}{3732480} & -\frac{13577}{1410877440} \\
1 & -\frac{1}{2} & -\frac{5}{18} & -\frac{61}{648} & -\frac{41}{1944} & -\frac{551}{155520} & -\frac{1999}{4199040} & -\frac{7519}{141087744} \\
1 & -\frac{1}{2} & -\frac{25}{72} & -\frac{95}{648} & -\frac{425}{10368} & -\frac{401}{46656} & -\frac{9685}{6718464} & -\frac{28423}{141087744} \\
1 & -\frac{1}{2} & -\frac{5}{12} & -\frac{91}{432} & -\frac{61}{864} & -\frac{16559}{933120} & -\frac{6659}{1866240} & -\frac{168689}{282175488}
\end{array}\right],
$$

$$
\mathbf{B}=\left[\begin{array}{rrrrrrr}
-\frac{14227}{180} & \frac{1446661}{1800} & -\frac{939433}{300} & \frac{5600291}{900} & -\frac{6060059}{900} & \frac{755413}{200} & -\frac{85603}{100} \\
\frac{9403}{210} & -\frac{88807}{210} & \frac{239049}{140} & -\frac{773939}{210} & \frac{155671}{35} & -\frac{298031}{105} & \frac{104677}{140} \\
-\frac{1354}{5} & \frac{10267}{10} & -\frac{1153}{2} & -2864 & 5924 & -\frac{44647}{10} & \frac{12253}{10} \\
\frac{10683}{5} & -\frac{67728}{5} & 36801 & -54876 & 47265 & -\frac{110988}{5} & \frac{22083}{5} \\
-1332 & 5148 & -3816 & -9792 & 20772 & -14652 & 3672 \\
-1080 & -2592 & 30456 & -74304 & 82296 & -44064 & 9288 \\
38880 & -241056 & 622080 & -855360 & 660960 & -272160 & 46656 \\
46656 & -279936 & 699840 & -933120 & 699840 & -279936 & 46656
\end{array}\right],
$$




$$
\mathbf{V}=\left[\begin{array}{rrrrrrrc}
1 & -\frac{12}{5} & -\frac{7}{2} & -2 & 0 & \frac{1352383}{2332800} & \frac{5051723}{13996800} & \frac{54761897}{440899200} \\
0 & 0 & -\frac{5}{2} & -\frac{485}{108} & -\frac{4873}{1296} & -\frac{227699}{116640} & -\frac{216487}{311040} & -\frac{16085969}{88179840} \\
0 & 0 & 0 & -\frac{25}{12} & -\frac{173}{54} & -\frac{11455}{5184} & -\frac{24713}{25920} & -\frac{1544617}{5598720} \\
0 & 0 & 0 & 0 & -\frac{5}{3} & -\frac{301}{144} & -\frac{1457}{1296} & -\frac{191087}{466560} \\
0 & 0 & 0 & 0 & 0 & -\frac{5}{4} & -\frac{497}{432} & -\frac{559}{1296} \\
0 & 0 & 0 & 0 & 0 & 0 & -\frac{5}{6} & -\frac{83}{216} \\
0 & 0 & 0 & 0 & 0 & 0 & 0 & -\frac{5}{12} \\
0 & 0 & 0 & 0 & 0 & 0 & 0 & 0
\end{array}\right] .
$$

For this method we have

$$
\begin{aligned}
& \eta=\left[\begin{array}{lllllll}
-\frac{7}{5760} & -\frac{587}{217728} & \frac{5239}{233280} & -\frac{3653}{77760} & \frac{1}{16} & \frac{101}{216} & 0
\end{array}\right]^{T}, \\
& \widetilde{\beta}=\left[\begin{array}{lllllll}
-\frac{190298051}{313528320} & -\frac{13949459}{100776960} & \frac{29848459}{78382080} & -\frac{51229}{373248} & -\frac{77453}{311040} & \frac{325}{864} & -\frac{19}{144}
\end{array}\right]^{T}, \\
& \xi=\left[\begin{array}{lllllll}
\xi_{1} & \xi_{2} & \xi_{3} & \xi_{4} & \xi_{5} & \xi_{6} & \xi_{7}
\end{array}\right]^{T} \\
& \xi_{1}=-\frac{5}{8064}, \quad \xi_{2}=-\frac{144266309}{203166351360}, \quad \xi_{3}=-\frac{2090489}{3174474240}, \quad \xi_{4}=-\frac{273361}{501645312}, \\
& \xi_{5}=-\frac{334847}{793618560}, \quad \xi_{6}=-\frac{12820033}{40633270272}, \quad \xi_{7}=-\frac{9181}{39191040}, \\
& \widetilde{\delta}=\left[\begin{array}{lllllll}
\frac{189730403}{313528320} & \frac{97500089}{705438720} & -\frac{28795867}{78382080} & \frac{6119}{69120} & \frac{97897}{311040} & -\frac{71}{288} & -\frac{181}{432}
\end{array}\right]^{T}, \\
& E=\frac{5455091477}{4702924800}, \quad F=-\frac{5457457409}{4702924800}, \\
& \varphi=\left[\begin{array}{lllllll}
-8024.10 & 9823.17 & 7881.06 & -3332.87 & -14874.3 & -14921.5 & 24222.4
\end{array}\right]^{T} \text {, } \\
& \psi=\left[\begin{array}{llllllll}
0 & -773.81 & -4469.3 & -3782.3 & -1850.1 & -582.7 & -134.9 & -24.83
\end{array}\right]^{T}, \\
& \bar{\varphi}=\left[\begin{array}{lllllll}
-47951.5 & 85060.4 & 33382.1 & -54938.6 & -89087.2 & -36688.2 & 115038.6
\end{array}\right]^{T} \text {, } \\
& \bar{\psi}=\left[\begin{array}{llllllll}
0 & -4815.49 & -22908.3 & -21151.7 & -10362.2 & -3199.6 & -724.9 & -130.7
\end{array}\right]^{T} \text {. }
\end{aligned}
$$

\section{Concluding Remarks}

In this paper we described a systematic search for highly stable GLMs with IQS. Examples of such methods of order $p=s+1=r$ and stage order $q=$ $p-1$ are presented up to the order $p=8$. These are the first examples of such methods of high order and stage order presented in the literature on the subject. Contrary to methods of low stage order, such as Runge-Kutta formulas, the GLMs constructed in this paper do not suffer from order reduction phenomenon [7]. This is a consequence of high stage order. The implementation of the methods will be the subject of future work, since the implementation issues require different tools than needed in the construction of methods. 


\section{Acknowledgements}

The authors wish to express their gratitude to anonymous referees for useful comments which helped to improve the presentation of the paper.

\section{References}

[1] Z. Bartoszewski and Z. Jackiewicz. Explicit Nordsieck methods with extended stability regions. Appl. Math. Comput., 218(10):6056-6066, 2012. http://dx.doi.org/10.1016/j.amc.2011.11.088.

[2] M. Braś. Nordsieck methods with inherent quadratic stability. Math. Model. Anal., 16(1):82-96, 2011. http://dx.doi.org/10.3846/13926292.2011.560617.

[3] M. Braś and A. Cardone. Construction of efficient general linear methods for non-stiff differential systems. Math. Model. Anal., 17(2):171-189, 2012. http://dx.doi.org/10.3846/13926292.2012.655789.

[4] M. Braś, A. Cardone and R. D'Ambrosio. Implementation of explicit Nordsieck methods with inherent quadratic stability. Math. Model. Anal., 18(2):289-307, 2013. http://dx.doi.org/10.3846/13926292.2013.785039.

[5] M. Braś and Z. Jackiewicz. Nordsieck methods with computationally verified algebraic stability. Appl. Math. Comput., 217(21):8598-8610, 2011. http://dx.doi.org/10.1016/j.amc.2011.03.096.

[6] M. Braś and Z. Jackiewicz. Search for efficient general linear methods for ordinary differential equations. J. Comput. Appl. Math, 262:180-192, 2014.

[7] J.C. Butcher. The Numerical Analysis of Ordinary Differential Equations. Runge-Kutta and General Linear Methods. John Wiley \& Sons Inc., Chichester, New York, 1987.

[8] J.C. Butcher. Diagonally-implicit multi-stage integration methods. Appl. Numer. Math., 11(5):347-363, 1993. http://dx.doi.org/10.1016/0168-9274(93)90059-Z.

[9] J.C. Butcher and Z. Jackiewicz. Diagonally implicit general linear methods for ordinary differential equations. BIT, 33(3):452-472, 1993. http://dx.doi.org/10.1007/BF01990528.

[10] J.C. Butcher and Z. Jackiewicz. Construction of diagonally implicit general linear methods of type 1 and 2 for ordinary differential equations. Appl. Numer. Math., 21:385-415, 1996. http://dx.doi.org/10.1016/S0168-9274(96)00043-8.

[11] J.C. Butcher and Z. Jackiewicz. Implementation of diagonally implicit multistage integration methods for ordinary differential equation. SIAM J. Numer. Anal., 34:2119-2141, 1997. http://dx.doi.org/10.1137/S0036142995282509.

[12] J.C. Butcher and Z. Jackiewicz. Construction of general linear methods with Runge-Kutta stability properties. Numer. Algorithms, 36(1):53-72, 2004. http://dx.doi.org/10.1023/B:NUMA.0000027738.54515.50.

[13] J.C. Butcher, Z. Jackiewicz and H.D. Mittelmann. A nonlinear optimization approach to the construction of general linear methods of high orders. J. Comput. Appl. Math., 81:181-196, 1997. http://dx.doi.org/10.1016/S0377-0427(97)00039-3.

[14] J.C. Butcher and W.M. Wright. The construction of practical general linear methods. BIT, 43(4):695-721, 2003.

http://dx.doi.org/10.1023/B:BITN.0000009952.71388.23. 
[15] A. Cardone and Z. Jackiewicz. Explicit Nordsieck methods with quadratic stability. Numer. Algorithms, 60:1-25, 2012. http://dx.doi.org/10.1007/s11075-011-9509-y.

[16] A. Cardone, Z. Jackiewicz and H.D. Mittelmann. Optimization-based search for Nordsieck methods of high order with quadratic stability. Math. Model. Anal., 17(3):293-308, 2012. http://dx.doi.org/10.3846/13926292.2012.685497.

[17] D. Conte, R. D'Ambrosio and Z. Jackiewicz. Two-step Runge-Kutta methods with quadratic stability functions. J. Sci. Comput., 44(2):191-218, 2010. http://dx.doi.org/10.1007/s10915-010-9378-x.

[18] D. Conte, R. D'Ambrosio, Z. Jackiewicz and B. Paternoster. Numerical search for algebraically stable two-step almost collocation methods. J. Comput. Appl. Math, 239(1):304-321, 2013. http://dx.doi.org/10.1016/j.cam.2012.08.012.

[19] G. Dahlquist. A special stability property for linear multistep methods. BIT, 3(1):27-43, 1963. http://dx.doi.org/10.1007/BF01963532.

[20] L.L. Hewitt and A.T. Hill. Algebraically stable general linear methods and the $G$-matrix. BIT, 49(1):93-111, 2009. http://dx.doi.org/10.1007/s10543-0080207-7.

[21] G. Izzo and Z. Jackiewicz. Construction of algebraically stable DIMSIMs. J. Comput. Appl. Math, 261:72-84, 2014. http://dx.doi.org/10.1016/j.cam.2013.10.037.

[22] Z. Jackiewicz. General Linear Methods for Ordinary Differential Equations. John Wiley \& Sons Inc., Hoboken, NJ, 2009.

[23] J. Schur. Über Potenzreihen die im Innern des Einheitskreises beschränkt sind. J. Reine Angew. Math., 147:205-232, 1916.

[24] W.M. Wright. The construction of order 4 DIMSIMs for ordinary differential equations. Numer. Algorithms, 26:123-130, 2001. http://dx.doi.org/10.1023/A:1016611914097.

[25] W.M. Wright. Explicit general linear methods with inherent Runge-Kutta stability. Numer. Algorithms, 31:381-399, 2002. http://dx.doi.org/10.1023/A:1021195804379.

[26] W.M. Wright. General linear methods with inherent Runge-Kutta stability. Doctoral thesis, The University of Auckland, New Zealand, 2002. 